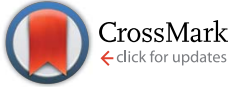

Cite this: RSC Adv., 2015, 5, 15379

Received 12th December 2014 Accepted 26th January 2015

DOI: $10.1039 / c 4 r a 16255 b$

www.rsc.org/advances

\title{
Preparing mono-dispersed liquid core PDMS microcapsules from thiol-ene-epoxy-tailored flow-focusing microfluidic devices
}

\begin{abstract}
P. Mazurek, ${ }^{a}$ A. E. Daugaard, ${ }^{a}$ M. Skolimowski, ${ }^{b}$ S. Hvilsted ${ }^{a}$ and A. L. Skov ${ }^{\star a}$
An applied dual-cure system based on thiol-ene and thiol-epoxy "click chemistry" reactions was proved to be an extremely effective and easy to use tool for preparing microfluidic chips, thereby allowing for precise control over material properties and providing the possibility of covalently bonding chip wafers. Different thiol-ene-epoxy-based polymer compositions were tested with the help of DSC and ATR FTIR, in order to investigate their physical and chemical properties. Water contact angles were determined, thus verifying the high efficiency and selectivity of the chemical surface modification of compositions in relation to high hydrophilicity and hydrophobicity. An obtained microfluidic device was subsequently used in order to produce PDMS microcapsules of very narrow size distribution and which contained various common liquids, such as water and ethanol, as well as an ionic liquid 2-hydroxyethylammonium formate.
\end{abstract}

\section{Introduction}

The importance of micro-encapsulation is undisputed, so significant effort is exerted nowadays to develop encapsulation techniques, although in many cases they are still far from optimal. Microcapsules, which by definition are solid or liquid spheres coated with a solid material separating the core from an outside medium, have great potential in the pharmaceutical, food, printing and coating industries, as well as in preparing engineering materials. ${ }^{1}$ Many methods have been developed allowing for the rather effective formation of core-shell spheres. Additionally, distinguishing between solid and liquid core microcapsules is necessary, since preparation procedures may in many cases vary and require very different techniques. The production of a liquid core structure is considered more complex than producing a solid core, and several parameters have to be taken into account, depending strictly on the technique chosen. When preparing liquid-core microcapsules special attention should be given to mini emulsion techniques, for example the phase separation method. Here, shell formation is induced through polymer precipitation caused by either the evaporation of a volatile solvent from vesicles containing polymer, solvent and the initial core material or through polymerisation that takes place within vesicles and causes the growing polymer to precipitate on the droplet surface. ${ }^{2-4}$ Shell

${ }^{a}$ Danish Polymer Centre, Department of Chemical and Biochemical Engineering, Technical University of Denmark, Building 227, DK-2800 Kgs. Lyngby, Denmark. E-mail: al@kt.dtu.dk

${ }^{b}$ Fluidic Array Systems and Technology, Department of Micro and Nanotechnology, Technical University of Denmark, Denmark formation can also take place in systems where one monomer is dissolved in continuous phase and the second monomer is dissolved in core material forming vesicles in an emulsion. Reactions between the two monomers, taking place on the emulsion interface, are another effective way of encapsulating liquids. ${ }^{5}$ These methods, although still in need of improvement, do not allow for full control over microcapsule dimensions and introduce many limitations concerning the choice of materials for use in these systems.

Modelling of the core-shell structure, which essentially means designing core material, and its size as well as shell thickness and physical and the chemical properties of the material allow for precise control over the release of core material as a result of, for example, shell degradation triggered by different factors such as UV-light, heat, $\mathrm{pH}$, etc. ${ }^{1}$ From this point of view, only one technique to date enables sufficient control over the encapsulation process and allows for preparing microcapsules extremely narrow in size distribution and tailored dimensions. This technique is based on flow-focusing microfluidic systems containing either a set of capillaries or a set of microchannel junctions of different wettability. Despite the fact that this method is still not used in mass production, we believe it is the most powerful tool for preparing core-shell microspheres on a laboratory scale. Many groups working on developing systems with improved efficiency and versatility have produced some fascinating results, all of which contribute to the increasing interest of material scientists in lab-on-chip techniques. ${ }^{6-9}$

In this paper we present a simple method for preparing a flow-focusing microfluidic device by applying thiol-ene click chemistry, which due to its great versatility and simple 
chemistry is being increasingly used for fabricating lab-on-chip devices. ${ }^{\mathbf{1 0 , 1 1}}$ This fascinating chemistry exhibits a great number of advantages, for example very high reaction efficiency, rapid reactions triggered by exposing compositions to UV-light, low exothermic effects and no byproducts. One of the greatest advantages of these systems is good solvent resistivity, which allows for working with almost any type of material and thereby making thiol-ene systems excellent replacements for the currently most widely used PDMS microfluidic chips. ${ }^{7}$ PDMS in some instances does not meet the requirements for lab-on-chip devices, for example poor shape consistency due to high flexibility of PDMS, very high gas permeability, rather poor solvent resistance against most organic solvents.

Thiol-ene chemistry is based on the reaction between vinylcontaining compounds and a mercapto-containing compound. In the first step, a thiyl radical is formed through hydrogen exchange between the thiol group and a radical source (Fig. 1). Subsequently the thiyl radical reacts rapidly with the vinyl group of the second compound, forming a thio-ether covalent bond and transferring the radical to the next thiol group. The reaction mechanism (Fig. 1) implies that only a minimal amount of photoinitiator is necessary for the reaction to progress and to reach maximum conversion. By applying different amounts of initiator and different UV-light intensities the reaction speed can be modified easily. Additionally, when $254 \mathrm{~nm}$ light is used, thiol-ene compositions with no photoinitiators can be crosslinked. ${ }^{12}$ In this approach we make use of the off-stoichiometry thiol-ene (abbreviated OSTE) composition presented by Carlborg et al., ${ }^{13}$ in which a tetrafunctional thiol compound is crosslinked with a trifunctional allyl compound, thus forming a stable matrix which exhibits good solvent resistance and possesses tunable mechanical and chemical properties. Selective surface patterning, necessary for preparing double emulsions in flow-focusing microfluidic systems, ${ }^{\mathbf{1 4}}$ is achieved by again applying thiol-ene chemistry, which in turn allows for efficient grafting onto polymer surfaces.

One of the greatest challenges when using flow-focusing microfluidic systems is the prevention of leakages appearing in chips when high pressure is present in microfluidic channels. It is especially intractable when higher-viscosity liquids are introduced to the system, since in such cases higher flow rates are necessary for the dispersed phase stream break-up to occur. Research on bonding strength between wafers made of different OSTE compositions was conducted by Sikanen et al. ${ }^{\mathbf{1 6}}$ All combinations of OSTE wafers presented by this group were

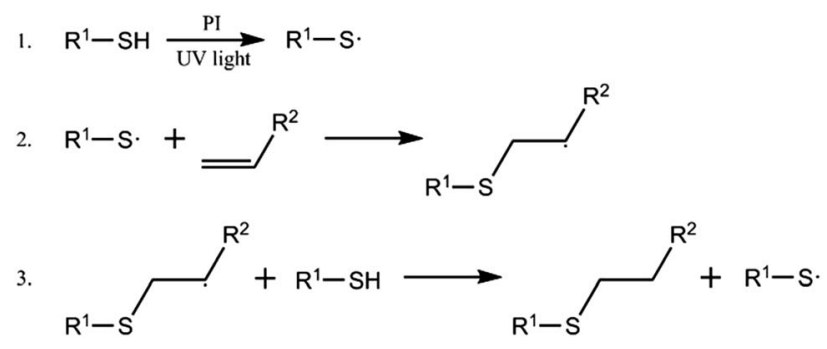

Fig. 1 Mechanism of a photoinitiated thiol-ene reaction. ${ }^{15}$ tested, but none of them appeared to provide adhesion strong enough to eliminate leakages completely, and hence a better method for wafer bonding was required. Herein, we modify a two-step crosslinking method presented by Saharil et al., ${ }^{17}$ which is based on the covalent bonding of the two microfluidic chip wafers through the introduction of an epoxy-containing compound working as a bonding agent. With the help of this method we managed not only to avoid leakages but also preserved the possibility of surface modification.

The flow-focusing microfluidic device presented in this study was designed in order to enable the microcapsule formation of any type of material, provided that the surface tension between the inner/outer phase and the middle phase is sufficiently high.

\section{Experimental}

\section{Materials and methods}

Two component Sylgard 184 silicone kit, used for fabricating mirror image moulds and for preparing single and double emulsions, was purchased from Dow Corning. Pentaerythritol tetrakis(3-mercaptopropionate) (PETMP), triallyl-1,3,5-triazine2,4,6(1H,3H,5H)-trione (TATATO) and (bisphenol A diglycidyl ether (BADGE)), forming a microfluidic chip matrix, as well as compounds used for grafting reactions (acrylic acid, allylmalonic acid (AMA), sodium hydroxide, 2,2,3,3,4,4,5,5,6,6,7,7dodecafluoroheptyl acrylate (DFHA), allyl trifluoroacetate (ATFA), allylpentafluorobenzene (APFB), $1 H, 1 H, 2 H$-perfluoro-1decene (PFD) and poly(ethylene glycol) methacrylate $\left(M_{\mathrm{n}}=\right.$ $360 \mathrm{~g} \mathrm{~mol}^{-1}$ ) (PEG), toluene, heptane) were purchased from Sigma Aldrich. Lucirin TPO-L and 2,2-dimethoxy-2phenylacetophenone (DMPA) photoinitiators were obtained from BASF GmbH Germany and Sigma Aldrich, respectively. The thiol-epoxy reaction catalyst 1,5-diazabicyclo-[4,3,0]-non-5ene (DBN) was purchased from Sigma Aldrich. Absolute ethyl alcohol, used for the grafting reaction, ionic liquid synthesis and the formation of a double emulsion, was supplied by Kemetyl A/S. Compounds used for forming single and double emulsions, namely sodium dodecyl sulfate (SDS) and isopropanol, were purchased from Sigma Aldrich, whereas polyvinyl alcohol (PVA) and glycerol were supplied by Fluka and Emmelev A/S, respectively. Formic acid and 2-hydroxyethylamine, used for synthesising ionic liquid, were purchased from J. T. Baker and Sigma Aldrich, respectively. Active carbon was provided by Sigma Aldrich. All chemicals were used as received.

A TA Instruments Differential Scanning Calorimeter Q1000 was used to determine the thermal behaviour of the samples. All tests were performed at a heating rate of $10{ }^{\circ} \mathrm{C} \mathrm{min}^{-1}$ across a temperature range of between $-30{ }^{\circ} \mathrm{C}$ and $120{ }^{\circ} \mathrm{C}$ in an $\mathrm{N}_{2}$ atmosphere. Reported glass transition temperatures of OSTE formulations were based on at least two independent measurements where deviations from individual tests were no higher than $2{ }^{\circ} \mathrm{C}$, whereas for OSTE+ formulations one measurement was conducted. Viscosities were measured with AR2000ex rheometer from TA Instruments. Nicolet iS50 FT-IR was used to obtain ATR FTIR spectra of neat and surfacemodified polymer compositions as well as synthesised ionic liquid. Water wettability of polymers was investigated with the 
<smiles></smiles>

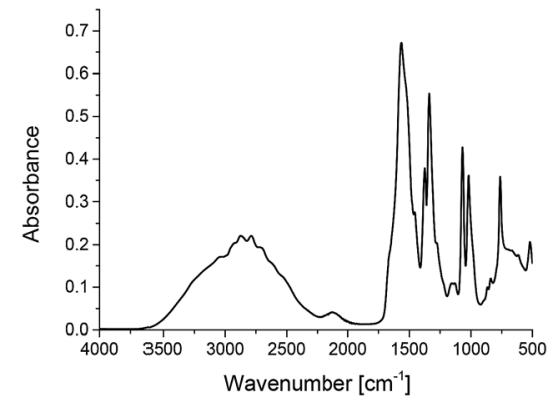

Fig. 2 Chemical formula (left) and ATR FTIR spectrum (right) of 2-hydroxyethylammonium formate, with a peak at a wavenumber of around $1565 \mathrm{~cm}^{-1}$, thereby indicating a $\mathrm{COO}^{-}$stretch, and a peak at a wavenumber of around $2125 \mathrm{~cm}^{-1}$, corresponding to an $\mathrm{RNH}_{3}{ }^{+}$bend.

help of DataPhysics OCA20. Each surface was tested using the sessile drop method, and the average water contact angle was calculated from at least three measurements. A Leica DM LB optical microscope was applied, in order to determine the morphology and sizes of microspheres and microcapsules produced.

\section{Synthesis of 2-hydroxyethylammonium formate}

An ionic liquid (IL) 2-hydroxyethylammonium formate (see chemical formula in Fig. 2) was synthesised following a procedure described by Yuan et al. ${ }^{18}$ In total, 0.2 mole of 2-hydroxyethylamine and formic acid were dissolved in $40 \mathrm{~mL}$ of ethanol. Formic acid solution was added drop-wise to a round-bottomed flask equipped with a magnetic stirrer and reflux condenser and containing 2-hydroxyethylamine solution. The reaction was held for around 4 hours to ensure full conversion. Subsequently obtained ionic liquid was separated from the solvent via vacuum evaporation, dissolved again in ethanol, treated with carbon black and then filtered. The product was kept under lowpressure conditions in order to avoid water condensation. The ATR FTIR spectrum of the ionic liquid confirmed an exchange of protons between Brønsted acid and the base, as shown in Fig. 2.

\section{Results and discussion}

\section{Preparing microfluidic device}

The microfluidic device contained two main units, each consisting of two parts. The microfluidic chip entails a bottom part,

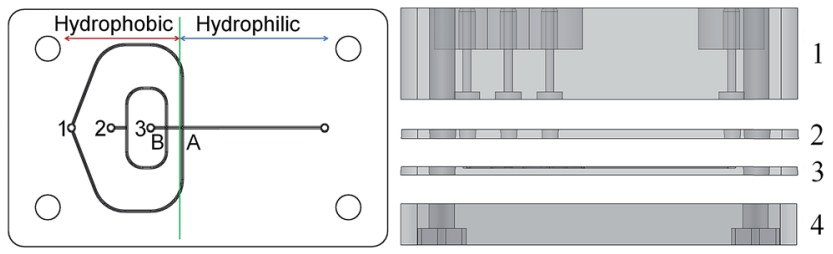

Fig. 3 Chip scheme (left) (channel width of $280 \mu \mathrm{m}$ ) and full microfluidic device (right). 1 - holder top, 2 - chip top, 3 - chip bottom, 4 - holder bottom.
1.

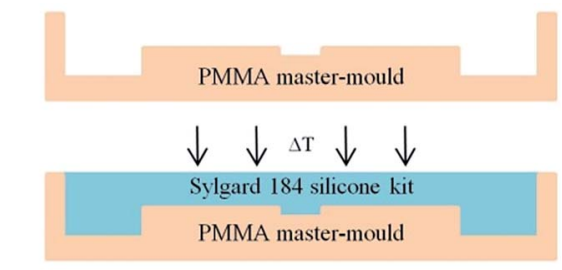

3.

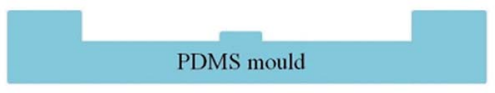

4.

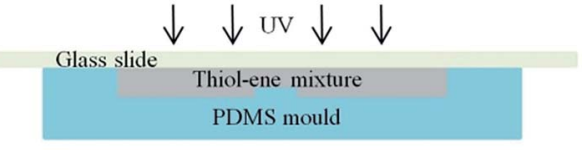

5.

Crosslinked thiol-ene network

Fig. 4 Thiol-ene chip fabrication process scheme.

with all channels and junctions, and a top part, which seals the chip. The three inlets and one outlet placed in the top part enable liquid transfer throughout the chip. The two wafers of the chip are assembled and sandwiched between bottom and top parts of the holder (see Fig. 3).

The holder wafers were micromilled in polycarbonate. The microfluidic chip was then prepared in a two-step replica moulding process, where all geometries of a master mould were micromilled in a PMMA plate in the first step. Subsequently a Sylgard 184 silicone kit (mixing ratio $10: 1$ ) composition was prepared, degassed and cast onto the PMMA master mould, in order to obtain a mirror image PDMS mould. The composition was then crosslinked at $80{ }^{\circ} \mathrm{C}$ for 10 hours, after which the mirror image mould was ready to be used for preparing the microfluidic chip. A full scheme for the thiol-ene chip fabrication process is presented in Fig. 4.

For preparing the microfluidic chip we employed the composition of monomers reported by Carlborg et al. ${ }^{13}$ The offstoichiometry thiol-ene composition (OSTE) is based on a tetrafunctional thiol compound and a tri-functional allyl compound (Fig. 5). The components form a stable network when mixed together with a photoinitiator and following subsequent exposure to UV-light. Depending on the stoichiometric amounts of both components, very different mechanical properties of the material can be obtained. Results for differential scanning calorimetry, presented in Fig. 6, show that when thiol and vinyl groups were used in stoichiometric balance, the glass transition temperature $\left(T_{\mathrm{g}}\right)$ of the composition reached a maximum $60^{\circ} \mathrm{C}$, which can be attributed to the highest degree of crosslinking in

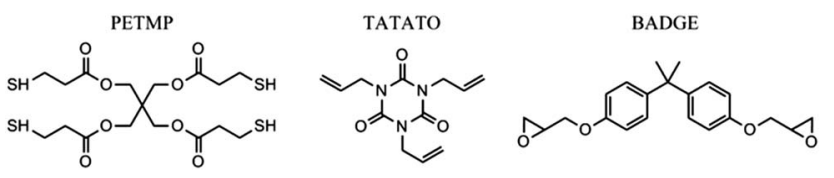

Fig. 5 Structural formulas of compounds used for preparing a flowfocusing microfluidic chip: tetrathiol (PETMP), triallyl (TATATO) and diepoxy (BADGE) working as a dual cure agent. 


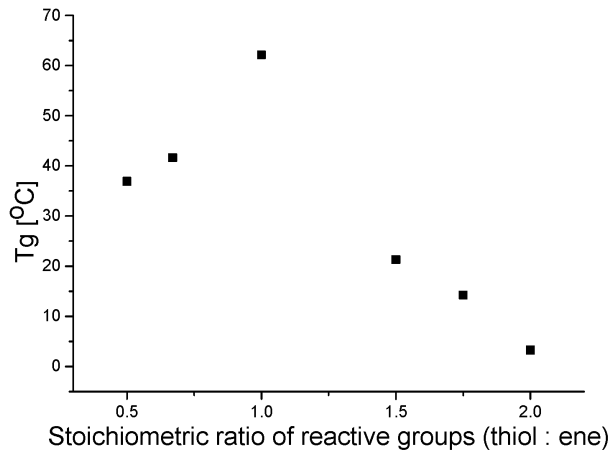

Fig. 6 Glass transition temperatures of different OSTE compositions.

this composition and the presumed absence of dangling chains. On the other hand, when stoichiometric imbalance is introduced, the glass transition temperatures decrease due to an increasing number of dangling chains with unreacted functional groups. It can also be hypothesised that excess triallyl monomer (TATATO) results in the formation of a stiffer structure than in the case of excess thiol monomer, which is finally attributed to the structure of monomers (shorter dangling chains and the cyclic nature of allyl monomer). The presence of both thiol and vinyl groups in different compositions was proven by FTIR tests, which for $r=2.0$ exhibited a peak at a wavenumber of around $2550 \mathrm{~cm}^{-1}$, attributed to the $\mathrm{S}-\mathrm{H}$ stretch of the thiol groups, and no peaks in the range between 3095 and $3010 \mathrm{~cm}^{-1}$, attributed to the $\mathrm{sp}^{2} \mathrm{C}-\mathrm{H}$ stretch of the vinyl group and vice versa in the case of compositions with excess allyl monomer (see Fig. 7).

Due to the fact that the thiol-ene reaction is initiated at the mercapto groups, a composition with excess thiol groups was chosen for fabricating microfluidic chips. It is believed that grafting allyl containing compounds onto thiol-containing surfaces provides higher grafting selectivity, since active thiyl radicals, when fixed permanently to the polymer surface, cannot migrate, and hence the grafting reaction will take place on the UV-exposed area exclusively, which is an essential feature for preparing double emulsions in flow-focusing systems. Nevertheless the composition used for preparing the chip did not provide sufficient adhesion between the chip wafers, which in turn caused leakages when pumping liquids through the

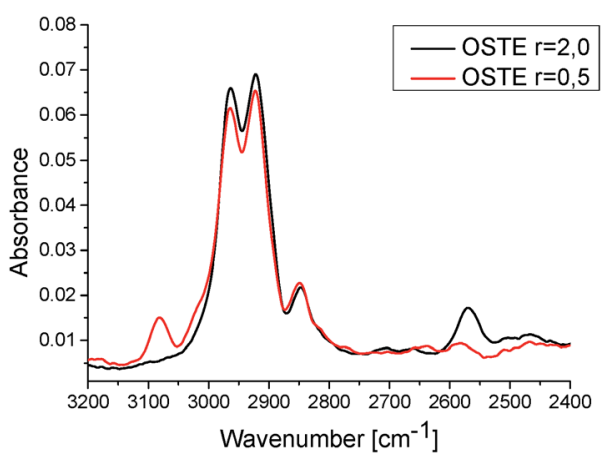

Fig. 7 FTIR spectra of OSTE compositions with $r=2.0$ and $r=0.5$.

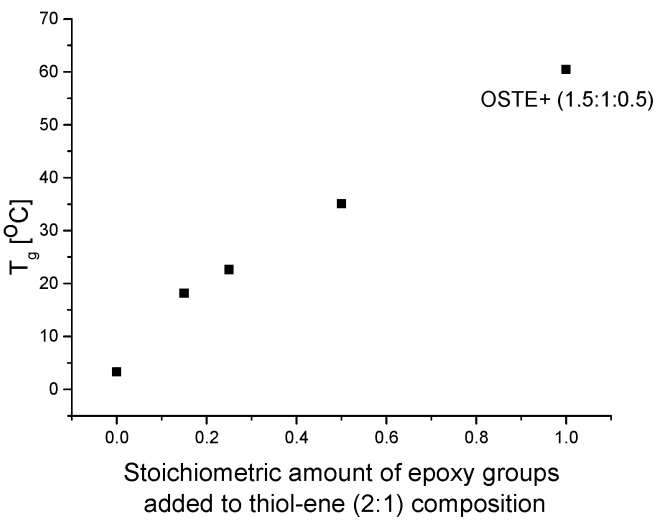

Fig. 8 Glass transition temperatures of different OSTE+ compositions (OSTE+ (1.5: $1: 0.5)$ as reference sample).

channels. Adhesion tests between different TATATO and PETMP compositions, as presented by Sikanen et al., ${ }^{16}$ reveal that the highest physical bond strength is achieved for formulations with excess allyl groups and for a combination of two different wafers (one wafer with excess allyl and one with thiol groups). For our application, none of the described systems showed sufficient adhesion, and therefore a third compound (BADGE) was introduced to the matrix, thereby making it a dualcure polymer composition - as described by Saharil et al. ${ }^{17}$ The new three-component formulation is abbreviated as OSTE+. In the dual-cure process a mixture of monomers was degassed, cast onto a PDMS mirror-image mould and UV-irradiated for a reaction between the thiol and allyl groups to take place. Afterwards, both chip wafers were demoulded and assembled together, thus avoiding the formation of air voids. No heating prior to demoulding was necessary due to the flexible nature of PDMS and the fact that the discussed OSTE+ composition after the UV-crosslinking step has $T_{\mathrm{g}}$ below room temperature. Therefore separation of chip wafers from the mould did not generate additional preparation steps as described by others. ${ }^{\mathbf{1 3}}$ The assembled wafers were then exposed to elevated temperatures $\left(80^{\circ} \mathrm{C}\right.$ for 2 hours), where the reaction between the epoxy groups and the remaining thiol groups took place both in the bulk as well as at the wafer-wafer interface. The thiol-epoxy reaction was catalysed by DBN, which was added to the system in minor amounts. Different reactive group molar ratios between PETMP, TATATO and BADGE were investigated and analysed in terms of DSC. Bonding strength between the two assembled chip wafers was determined from organoleptic observations. Glass transition temperatures of the tested formulations showed increasing values in line with the increasing addition of BADGE into the initial thiol-ene composition, which was kept at a constant thiol/allyl ratio of $r=$ 2.0 (see Fig. 8). We observed that by adding a small amount of BADGE (stoichiometric ratio of thiol : allyl : epoxy groups $2: 1: 0.15$ ), the glass transition temperature increased from around $2{ }^{\circ} \mathrm{C}$ to almost $20{ }^{\circ} \mathrm{C}$ and further to around $25^{\circ} \mathrm{C}$ and 35 ${ }^{\circ} \mathrm{C}$ for samples with stoichiometric ratios of $2: 1: 0.25$ and $2: 1: 0.5$, respectively. As a reference point, a composition with molar ratio of $1.5: 1: 0.5$, as reported by Saharil, ${ }^{17}$ was prepared 


\begin{tabular}{ll|lr}
\hline & & \\
\hline
\end{tabular}

Fig. 9 Wetting properties of $2: 1: 0.15$ OSTE+ samples grafted with different compounds in terms of static contact angle.

and also tested in terms of differential scanning calorimetry. As expected, the glass transition temperature of this formulation showed the highest value (around $60^{\circ} \mathrm{C}$ ), due to the fact that all functional groups reacted and left no dangling chains in bulk. Analogously, an increase in stiffness was observed in line with an increasing amount of BADGE in the system, followed by improved bonding strength between the wafers. For our purposes optimal properties were obtained for the $2: 1: 0.15$ OSTE + formulation, which showed improved adhesion between the wafers and additionally provided the highest amount of unreacted thiol groups on the surface - an important factor for further surface patterning.

As presented in Fig. 3, in order to prepare a water-oil-water emulsion it is desirable to create a microfluidic chip with one hydrophobic and one hydrophilic junction. To achieve this aim, thiol groups remaining on the surface of the OSTE+ composition react with different vinyl containing compounds, following which their influence on surface wettability is investigated. Surface patterning follows the same thiol-ene reaction mechanism, and therefore if more accurate surface modification is desirable, it is more effective to graft vinyl-containing compounds onto thiol-containing surfaces - and not vice versa. The grafting reactions were performed on the OSTE+ films by immersing them in $10 \mathrm{wt} \%$ solutions of different vinyl compounds in absolute ethanol along with the addition of DMPA as a photoinitiator. Reactions were carried out at a UV-light $(365 \mathrm{~nm})$ intensity of around $4 \mathrm{~mW} \mathrm{~cm}^{-2}$ for 1520 minutes, to ensure the full conversion of thiol groups. It has been reported, that the solvent-mediated thiol-ene grafting reaction can be accelerated significantly by applying UV-light of higher intensities. ${ }^{19} \mathrm{~A}$ series of grafting reactions was performed in order to reveal the most suitable chemicals that would effectively and permanently change the wetting properties of the material to the highest possible levels of hydrophobicity and
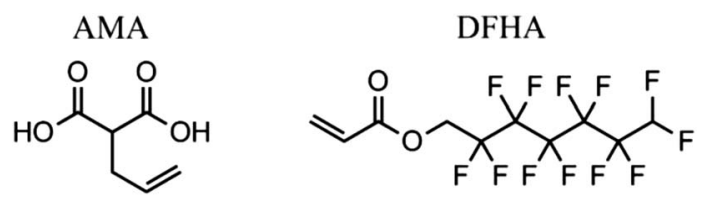

Fig. 10 Structural formulas of allylmalonic acid 2,2,3,3,4,4,5,5,6,6,7,7-dodecafluoroheptyl acrylate. hydrophilicity, respectively. Different grafting conditions were additionally tested in order to determine the most efficient grafting procedure. Experiments showed that increasing the amount of photoinitiator, from 0.5 to $3 \mathrm{wt} \%$, did not improve grafting efficiency. Furthermore, Lucirin TPO-L proved to be an effective photoinitiator for grafting reactions when ethanol and isopropanol were used as reaction media. Different solvents (e.g. ethanol, isopropanol, toluene, heptane) used for surface modification reactions showed comparable contact angles albeit with one exception, where it was found that acrylic compounds tend to homopolymerise when toluene is used as a solvent. After just 3-4 minutes of UV-irradiation, a grafting solution changed colour from transparent to white, which indicates the progressing homopolymerisation of acrylic monomers. Increased concentrations of grafted compounds also did not improve grafting efficiency in the investigated range. We obtained same water contact angles for concentrations between 3 and $10 \mathrm{wt} \%$. Results of the experiments, supported by static contact angle measurements, are presented in Fig. 9, and the structural formulas of the two grafted compounds that alter the surface properties most efficiently are shown in Fig. 10. The best results regarding obtaining the highest hydrophilicity were acquired through grafting AMA. The grafted surface was subsequently flushed with a $0.01 \mathrm{M} \mathrm{NaOH}$ solution, performed in order to replace the protons of AMA carboxyl groups with sodium cations, which resulted in a significant increase in hydrophilicity. The highest hydrophobicity was obtained for $2: 1: 0.15$ OSTE + grafted with DFHA, where the static contact angle increased from $74.1^{\circ}$ for a neat sample to $100.6^{\circ}$ for a modified sample. This value could be increased even further by grafting DFHA onto a $2: 1: 0.25$ OSTE+ sample, which showed a static contact angle of $106.3 \pm 0.4^{\circ}$

Surface selectivity of the grafting reactions was finally proved by performing two-step surface patterning. The OSTE+ polymer film was first immersed in the DFHA ethanol solution with the addition of DMPA, while half of the film was covered with a stencil mask (black rubber film), thus preventing it from reacting when the sample was subsequently exposed to UV-light. After the first surface modification step was complete, the polymer film was thoroughly washed with a substantial amount of ethanol and blow-dried with $\mathrm{N}_{2}$. In the second step the sample was immersed in AMA solution but this time covering the already grafted half with the stencil mask and exposed to UV-light (a schematic illustration of the full process is in Fig. 11). Afterwards the polymer film was cleaned again, flushed with $0.01 \mathrm{M} \mathrm{NaOH}$ solution and then blow-dried. Static contact angle tests performed on both sides of the sample proved the validity of the two-step grafting process, by providing contact angle values comparable to results obtained from the simple surface modification process. The two-step surface patterning process was applied to the modification of the microfluidic chip channel surfaces, though the grafting reactions took place within chip channels exclusively after both wafers were covalently bonded.

The progress of the grafting reactions was monitored by ATR FTIR. It was shown that the grafting reaction of small molecules takes place not only on the surface of the material but also, to 


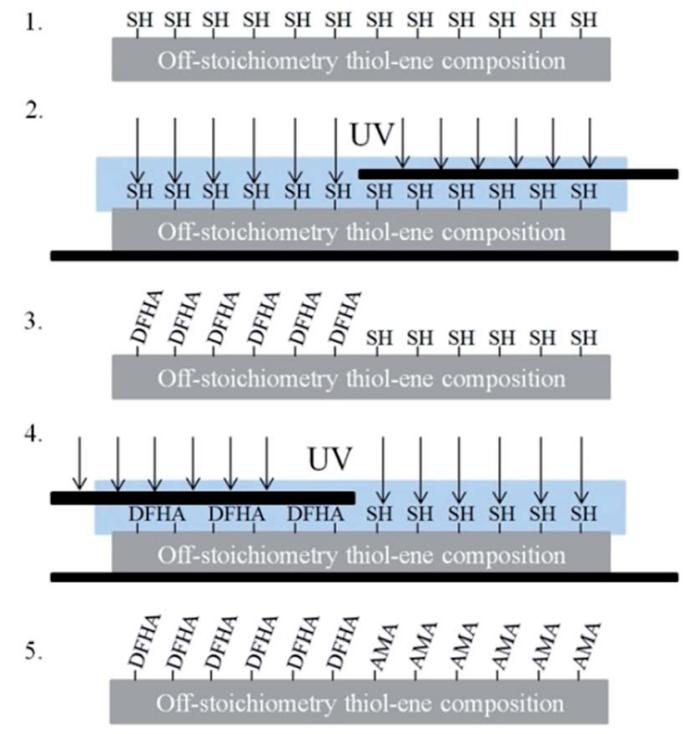

Fig. 11 Schematic illustration of the two-step surface patterning of the OSTE+ polymer film. SH represents unreacted thiol groups present on the polymer surface that subsequently react with DFHA and AMA in separate steps of the surface patterning process.

some extent, in bulk, which is attributed to the fact that the polymer network contains a substantial amount of dangling chains due to the relatively high off-stoichiometry ratio of the initial composition. The FTIR analyser beam usually penetrates through the first few microns of tested samples, so the spectra obtained should - in principle - show a small peak for the thiol group at the wavenumber of $2550 \mathrm{~cm}^{-1}$, as it is in the case of the initial network. Spectra obtained, for example, for OSTE+ grafted with sodium acrylate show no peaks in that region. Additionally, other strong peaks are to be observed at different wavenumbers, for example stretches typical for carboxylic salts at around $1565 \mathrm{~cm}^{-1}$ and $1425 \mathrm{~cm}^{-1}$ (Fig. 12), which was shown by Gonzalez et al. ${ }^{20,21}$ In the case of grafting larger molecules, such as DFHA, there are no significant changes in FTIR spectra, although contact angle measurements show substantial divergence in wetting properties.

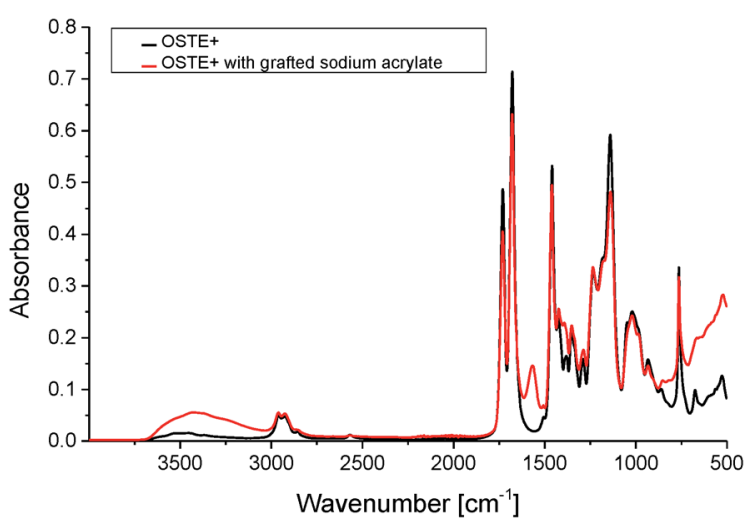

Fig. 12 ATR FTIR spectra of OSTE+ and OSTE+ grafted with sodium acrylate, presenting changes in absorbance at wavenumbers of $2550 \mathrm{~cm}^{-1}, 1565 \mathrm{~cm}^{-1}$ and $1425 \mathrm{~cm}^{-1}$.

\section{Formation of a single emulsion}

Separately controlled Harvard Apparatus 11 plus syringe pumps were used to introduce liquids to the microfluidic chip. Formation of a single emulsion was performed using inlets 1 and 2. Inlet 3 was plugged, which consequently blocked all liquid transfers through this connector (see Fig. 3). Two component Sylgard 184 silicone kit mixed to a ratio of $3: 2$ (base : crosslinker), was used as the dispersed phase $(\eta=1080$ $\mathrm{mPa} s$ at shear rate of $0.1 \mathrm{~s}^{-1}$ ) and injected into the system through inlet 2, while SDS/PVA aqueous solution (3 $\mathrm{wt} \%$ and $1 \mathrm{wt} \%$, respectively) was used as a carrier phase and was injected through inlet $1\left(Q_{1}>Q_{2}\right.$, where $Q$ corresponds to the flow rates of liquids). Droplet formation took place at junction A. Initial flow rates for each experiment were $10 \mathrm{~mL} \mathrm{~h}^{-1}$ and 1 $\mathrm{mL} \mathrm{h}^{-1}$ for $Q_{1}$ and $Q_{2}$, respectively (initial droplet diameter was $250 \mu \mathrm{m}$ ). Depending on liquid flow rates single emulsions of different droplet sizes (between 100 and $425 \mu \mathrm{m}$ ) and very narrow size distributions were obtained (see Fig. 13). In order to obtain smallest droplet size, the outer phase was injected at a flow rate of $50 \mathrm{~mL} \mathrm{~h}^{-1}$. By increasing the flow rate even higher to $100-200 \mathrm{~mL} \mathrm{~h}^{-1}$ the size distribution of spheres was significantly broadened. Nonetheless no leakages were observed. As an alternative method for altering droplet diameters, different carrier phase compositions were applied. Modifying the viscosity of the outer phase, which in this case could be done by increasing surfactant concentrations, works in favour of decreasing sphere sizes. As a result the range of sphere sizes can be shifted towards lower diameters. This effect can be achieved, for example, by increasing the amount of PVA, which substantially influences the viscosity of the solutions.

\section{Formation of a double emulsion}

Three liquids have to be introduced to the microfluidic chip in order to obtain core-shell microspheres. In this study we present tests where deionised water, ionic liquid ((2-hydroxyethyl)ammonium formate), ethanol, isopropanol and different water-glycerol mixtures were used as the inner phase of double emulsions. In each experiment a Sylgard 184 silicone kit, mixed to a weight ratio of $3: 2$ (base : crosslinker), was used as the middle phase and water with various surfactants as the outer phase.

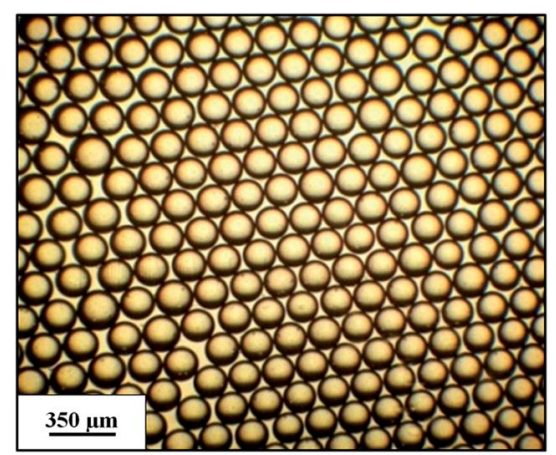

Fig. 13 Microscope image of silicone in water single emulsion obtained on junction A. 


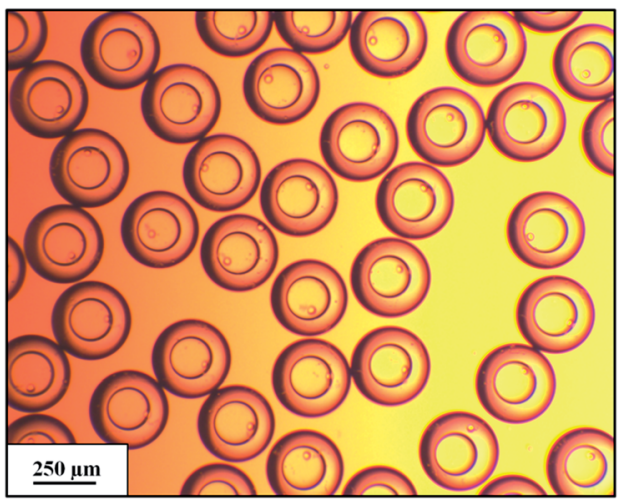

Fig. 14 Optical microscopy image of a water-in PDMS-in water double emulsion.

In the first experiment water was used as the inner phase of the double emulsion and SDS, PVA water solutions (3 $\mathrm{wt} \%$ and $1 \mathrm{wt} \%$, respectively) as the outer phase. In the first step the outer phase liquid is introduced to the chip via inlet 1 (see Fig. 3), followed by introducing the middle phase $\left(Q_{1}>Q_{2}\right)$. Initial flow rates were $Q_{1}=10 \mathrm{~mL} \mathrm{~h}^{-1}$ and $Q_{2}=1 \mathrm{~mL} \mathrm{~h}^{-1}$. As a result silicone droplet formation takes place at junction $\mathrm{A}$ and, as in the single emulsion case described paragraph above, droplets size is determined by the outer phase liquid flow rate. After a stable and mono-dispersed single emulsion is obtained, the inner phase is introduced to the chip via inlet $3\left(Q_{1}>Q_{2}>Q_{3}\right)$. $Q_{3}$ is slowly increased until the desired core-shell morphology with designed core and shell dimensions is obtained. The resulting double emulsion is collected via the outlet of the microfluidic chip and then left for the crosslinking reaction of the PDMS shell to take place. We confirm that, as in case of low viscosity liquids, ${ }^{8,22}$ the higher viscosity liquids investigated in this approach can also be used successfully in flow-focusing microfluidic chips. Additionally in these systems, core-shell microsphere dimensions can also be altered by varying the flow rates of injected liquids. Results of encapsulating water within a PDMS shell can be seen in Fig. 14.

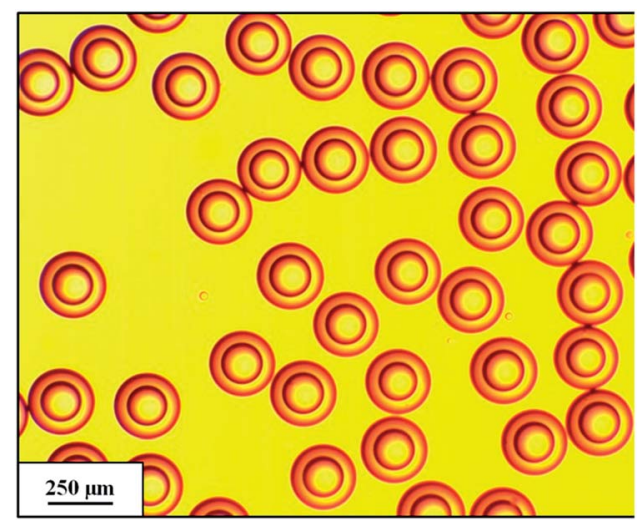

Fig. 15 Optical microscopy image of a 2-hydroxyethylammonium formate-in PDMS-in water double emulsion.

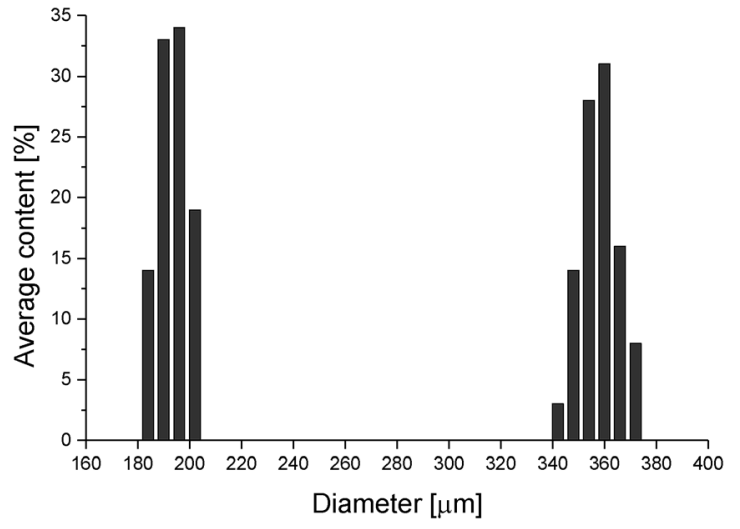

Fig. 16 An example of a size distribution of capsule cores (left columns) and capsules (right columns) obtained from the water-in PDMS-in water double emulsion.

The 2-hydroxyethylammonium formate was then used as a core material for the microcapsules, where PDMS was used as a shell material. The process for preparing the microcapsules followed the same scheme as in the water encapsulation case, whereby injection of the outer phase was followed by injection of the middle and then inner phases. An example of an acquired double emulsion can be seen in Fig. 15. Again, microcapsules of narrow size distribution were obtained, which proves that the flow-focusing microfluidic system we present in this study is a versatile method of encapsulation and can be applied to a broad range of materials. It was observed that by using IL (reported viscosity $\eta=118 \mathrm{mPa} s$ (ref. 18)), microcapsules of smaller diameters in comparison to water-core spheres were obtained. Attempts to encapsulate ethanol, isopropanol and different water and glycerol mixtures within a PDMS shell also resulted in the formation of microcapsules of very narrow size distribution, which again proves the versatility of the microfluidic system.

Size distribution of spheres in single and double emulsions was obtained by measuring diameters of at least 100 droplets. An average size and standard deviation were determined for each experiment. That allowed for calculations of coefficients of variation $(\mathrm{CV})$, which were proved to be held at substantially low values $(\mathrm{CV}<3 \%)$ for all experiments. An example of size distribution of water-in PDMS-in water emulsion is presented in Fig. 16.

The curing process for core-shell microspheres has been tested and optimised example water/PDMS/water double emulsion. The acquired double emulsion was collected into a beaker containing a water solution of SDS and PVA ( $3 \mathrm{wt} \%$ and $1 \mathrm{wt} \%$, respectively) under constant stirring with a magnetic stirrer, which prevents the double emulsion from forming microcapsule aggregates. The collected capsules were left for 24 hours at room temperature for the PDMS composition to be crosslinked and then form a solid shell. Increasing the temperature at this stage effectively accelerates the curing process, although too high a temperature results in disruption to the double emulsion and causes a collapse in microcapsules. The solution containing solid-shell capsules was heated up to $70{ }^{\circ} \mathrm{C}$ to ensure full crosslinking of the material. 
After each use, the microfluidic chip was thoroughly flushed with soap water, ethanol and heptane, and then once again with ethanol in order to remove possible PDMS residues and other impurities from the chip channels. This allows for the multiple use of a single chip and maintains surface properties, even up to several months and potentially longer. Experiments show that flushing the channels with soap water and ethanol only significantly lowers the lifetime of the chip. This appears to be a big advantage of this system over microfluidic devices based on PDMS, where surface properties and wafer bonding are usually achieved through plasma treatment, thereby causing temporary effects only.

\section{Conclusions}

A flow-focusing microfluidic device based on thiol-ene "click" chemistry was designed and fabricated. Due to the applied chemistry, physical and chemical properties of the chip could be adjusted to the intended application of producing elastomeric microcapsules. As a result it was possible to modify chemically the microfluidic chip channel surfaces in a controlled way and additionally to prevent any leakages while pumping liquids through the channels at very high flow rates. The developed system is an efficient tool for preparing core-shell microspheres of extremely narrow size distribution with tunable core diameters and shell thicknesses. We have demonstrated that very different liquids can be encapsulated within a PDMS shell where the only limitations are surface tension between liquids and their viscosities. The developed microfluidic device additionally has a very long lifespan, which efficiently saves time when conducting experiments on double emulsions. The versatility of the design creates a robust basis for fabricating new flow-focusing microfluidic devices for forming any type of core-shell microspheres for various applications. We believe that encapsulating liquid substances within soft PDMS shells is a great step towards selfhealing elastomeric materials.

\section{Acknowledgements}

The authors acknowledge funding from Innovationsfonden Denmark.

\section{Notes and references}

1 A. Latnikova, D. O. Grigoriev, H. Möhwald and D. G. Shchukin, J. Phys. Chem. C, 2012, 116, 8181-8187.
2 L. Gonzalez, M. Kostrzewska, M. Baoguang, L. Li, J. H. Hansen, S. Hvilsted and A. L. Skov, Macromol. Mater. Eng., 2014, 299, 1259-1267.

3 E. B. Murphy and F. Wudl, Prog. Polym. Sci., 2010, 35, 223251.

4 B. J. Blaiszik, N. R. Sottos and S. R. White, Compos. Sci. Technol., 2008, 68, 978-986.

5 V. Mittal, Encapsulation Nanotechnologies, John Willey \& Sons, Inc., Hoboken, 2013.

6 T. Nisisako, S. Okushima and T. Torii, Soft Matter, 2005, 1, 23-27.

7 G. T. Vladisavljević, I. Kobayashi and M. Nakajima, Microfluid. Nanofluid., 2012, 13, 151-178.

8 N. N. Deng, Z. J. Meng, R. Xie, X. J. Ju, C. L. Mou, W. Wang and L. Y. Chu, Lab Chip, 2011, 11, 3963-3969.

9 W. A. C. Bauer, M. Fischlechner, C. Abell and W. T. S. Huck, Lab Chip, 2010, 10, 1814-1819.

10 A. B. Lowe, Polym. Chem., 2010, 1, 17-36.

11 C. E. Hoyle and C. N. Bowman, Angew. Chem., Int. Ed., 2010, 49, 1540-1573.

12 N. B. Cramer, S. K. Reddy, M. Cole, C. Hoyle and C. N. Bowman, J. Polym. Sci., Part A: Polym. Chem., 2004, 42, 5817-5826.

13 C. F. Carlborg, T. Haraldsson, K. Öberg, M. Malkoch and W. Wijngaart, Lab Chip, 2011, 11, 3136-3147.

14 W. C. Joeng, M. Choi, C. H. Lim and S. M. Yang, Lab Chip, 2012, 12, 5262-5271.

15 J. A. Carioscia, J. W. Stansbury and C. N. Bowman, Polymer, 2007, 48, 1526-1532.

16 T. M. Sikanen, J. P. Lafleur, M. E. Moilanen, G. Zhuang, T. G. Jensen and J. P. Kutter, J. Micromech. Microeng., 2013, 23, 037002.

17 F. Saharil, F. Forsberg, Y. Liu, P. Bettotti, N. Kumar, F. Niklaus, T. Haraldsson, W. Wijngaart and K. B. Gylfason, J. Micromech. Microeng., 2013, 23, 025021.

18 X. L. Yuan, S. J. Zhang and X. M. Lu, J. Chem. Eng. Data, 2007, 52, 595-599.

19 G. Pardon, F. Saharil, J. M. Karlsson, O. Supekar, C. F. Carlborg, W. Wijngaart and T. Haraldsson, Microfluid. Nanofluid., 2014, 17, 773-779.

20 L. Gonzalez, A. L. Skov and S. Hvilsted, J. Polym. Sci., Part A: Polym. Chem., 2013, 51, 1359-1371.

21 L. Gonzalez, A. L. Skov and S. Hvilsted, Macromol. Symp., 2014, 342, 8-20.

22 F. Chang and Y. Su, J. Micromech. Microeng., 2008, 18, 065018. 